\title{
A 76-77GHz PULSED-DOPPLER RADAR MODULE FOR AUTONOMOUS CRUISE CONTROL APPLICATIONS
}

\author{
I. Gresham, N. Jain ${ }^{\dagger}$, T. Budkat ${ }^{\dagger t}$ A. Alexanian ${ }^{\dagger \dagger}$, \\ N. Kinayman, B. Ziegner, S. Brown, P. Staecker ${ }^{\dagger t t}$ \\ M/A-COM, LOWELL, MA \\ 'INDIAN INSTITUTE OF SCIENCE, BANGALORE, INDIA \\ "RF MICRO DEVICES BOSTON DESIGN CENTER, BILLERICA, MA \\ "H167 CEDAR ST., LEXINGTON, MA
}

\begin{abstract}
A single-substrate radar transceiver module suitable for 76-77GHz pulsed-Doppler applications has been developed. The packaged transceiver, including three waveguide ports and IF output, measures $20 \times 22 \times$ $8 \mathrm{~mm}$. The circuit is realized using discrete GaAs/AIGaAs pHEMTs, GaAs Schottky diodes and varactor diodes, as well as GaAs PIN and PHEMT MMICs mounted on a low-cost $127 \mu \mathrm{m}$ thick glass substrate.
\end{abstract}

\section{INTRODUCTION}

The field of radar for automotive applications and other mm-wave sensors is attracting great interest and investment [1]. The focus to date has been hardware demonstrations that fulfill the required functionality [2], [3]. Currently available Autonomous Cruise Control (ACC) radar systems are unlikely to meet the projected cost profile increased consumer take-up requires.

With projected demands of millions of units a year, the problem of high-volume, low-cost, highyield manufacturing and testing of these systems has not been adequately addressed beyond the development of MMIC building blocks [4-7], although recognition of the issue is increasing [8]. Even with these MMICs, the problem of integration with a circuit medium, packaging, and providing other mechanical and electrical interfaces remains a technically challenging and potentially costly issue [9]. A successful resolution of this issue requires a design approach that considers not only the electrical functionality, but also the means of integration and packaging and, perhaps more importantly, the compatibility with automated high-volume manufacturing techniques [10], [11].

The radar transceiver described in this paper was designed using several novel techniques to address and resolve these issues. The module principally consists of low-cost, high-yield discrete devices that are mounted using conventional and proven assembly techniques onto a glass substrate. The novel packaging technique allows for rapid interchangeability of mm-wave modules within each radar system, facilitating test. The module presented in this paper, along with all of the separately tested individual components, met specification on the first attempt.

\section{TRANSCEIVER COMPONENTS AND ARCHITECTURE}

The radar transceiver is realised by mounting discrete active devices and MMICs onto a glass substrate. Discrete devices are used in preference to MMICs wherever possible for two reasons; firstly, the yield and relative cost/unit area of a discrete device is significantly lower than that of a MMIC. Secondly, the designer is not restricted to the limited number of device types typically available in a given MMIC process. Semiconductor devices are mounted by flip-chip attach for the varactor and Schottky diodes, and using normal and compensated wire-bond interconnects for the PHEMTs and MMICs.

The glass substrate is realized using M/ACOMs proprietary passive GMIC process [12], [13]. 
Silicon and glass are combined to create a low-cost circuit medium that has attractive properties for millimeter-wave design. In this instance, the substrate contains low-loss transmission lines and biasing networks as well as providing a low-loss microstrip to waveguide interface by means of a special mode transducer and launch network. Microstrip, coplanar waveguide, lumped and distributed circuit elements and isolating RF ground shields can and have been utilized wherever appropriate for the circuit design. The components can be placed either flip-chip or upright using standard automated high-volume manufacturing equipment. All bondwire interconnects are designed to be implemented with standard $25 \mu \mathrm{m}$ diameter ball-bonds. A photograph of the complete transceiver module is shown in Figure 1.

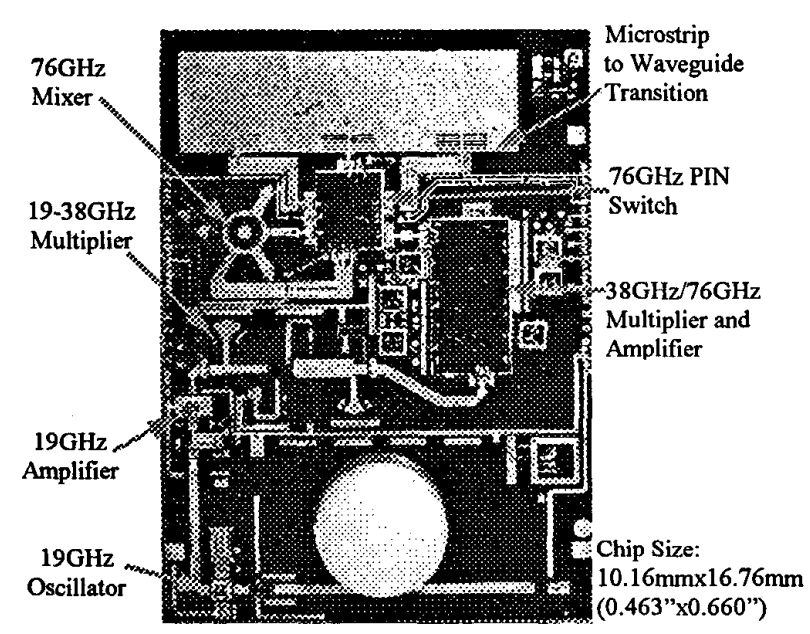

Figure 1 - The complete $76-77 \mathrm{GHz}$ transceiver module as realized using a glass (GMIC) substrate

The ACC system is a switched three-beam pulse-Doppler radar operating at $76-77 \mathrm{GHz}$. Figure 2 illustrates the module block diagram employed. The output from a voltage controlled oscillator (VCO) at $19 \mathrm{GHz}$, thermally stabilized by a dielectric puck, is amplified and multiplied to $38 \mathrm{GHz}$. The VCO, amplifier and multiplier are based on upright bonded discrete PHEMTs and flip-chip mounted varactor diodes. The $38 \mathrm{GHz}$ signal is then further multiplied and amplified to provide an output signal at $76 \mathrm{GHz}$.

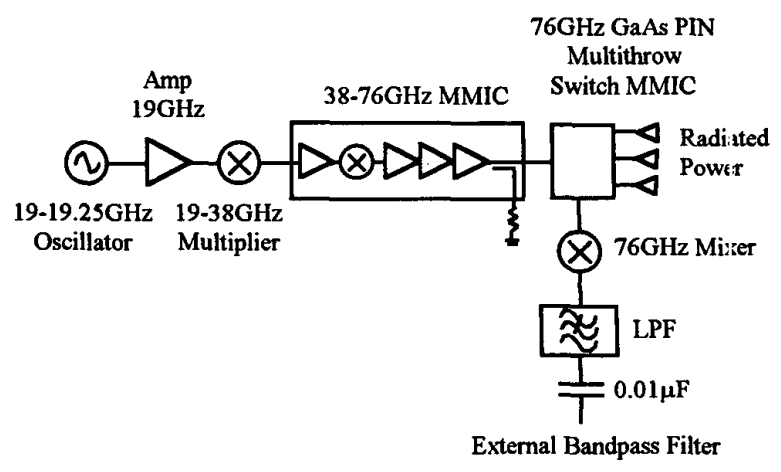

Figure 2 - Circuit block diagram of the $76 \mathrm{GHz}$ transceiver midule

An alternative configuration to that described that further reduces the area of active semiconductor material required, and thus the cost, is to replace the $38-76 \mathrm{GHz}$ MMIC by a $38 \mathrm{GHz}$ driver-amplifier and a second passive multiplier. The passive varactor-diode multiplier circuit needs careful design for thermal management of the power dissipated in the varactor junction.

When the module is in transmit mode (TX), the $76 \mathrm{GHz}$ signal is directed via the PIN switch MMIC to one of three antenna feeds providing azimuth object detection. This switch is a molified version of the reflective GaAs PIN switch described by Putnam et al, that is capable of being RF rested with a six-port test system for providing known good die [14]. In receive mode (RX), the $19 \mathrm{GHz} \mathrm{VCO}$ is tuned to an appropriate IF offset and the $76 \mathrm{GHz}+\mathrm{IF}$ is switched to the LO arm of the mixer. The return signal received by the antenna is then heterodyne mixed with the LO and the IF recovered.

\section{MODULE ASSEMBLY AND MANUFACTURING}

The aggressive price targets of the consumer marketplace demands that not only should the material costs of the millimeter-wave circuity be minimized, but that assembly should be autonated, and the design be insensitive to typical manufaciuring and device tolerance variation to maximize yield. Furthermore, the integration and test of the completed 
module should be possible without complex and expensive alignment or manual re-work procedures.

The glass substrate is mounted on a metal header that forms the package base and also the mounting surface for the three-waveguide feeds. The mm-wave interface between the transceiver module and the waveguide feeds is a tapered waveguide channel fed from a patent pending microstrip to waveguide mode transition on the front-side of the glass substrate. The metal header is compatible with standard high volume metal-injection-molding (MIM) technology. The glass circuit can be attached to the metal header using pick-and-place technology. The transition between the planar circuit on the front-side of the substrate and the waveguide-feeds in the base of the package is capable of misalignment by up to $200 \mu \mathrm{m}$ providing a robust design for variation in placement in assembly.

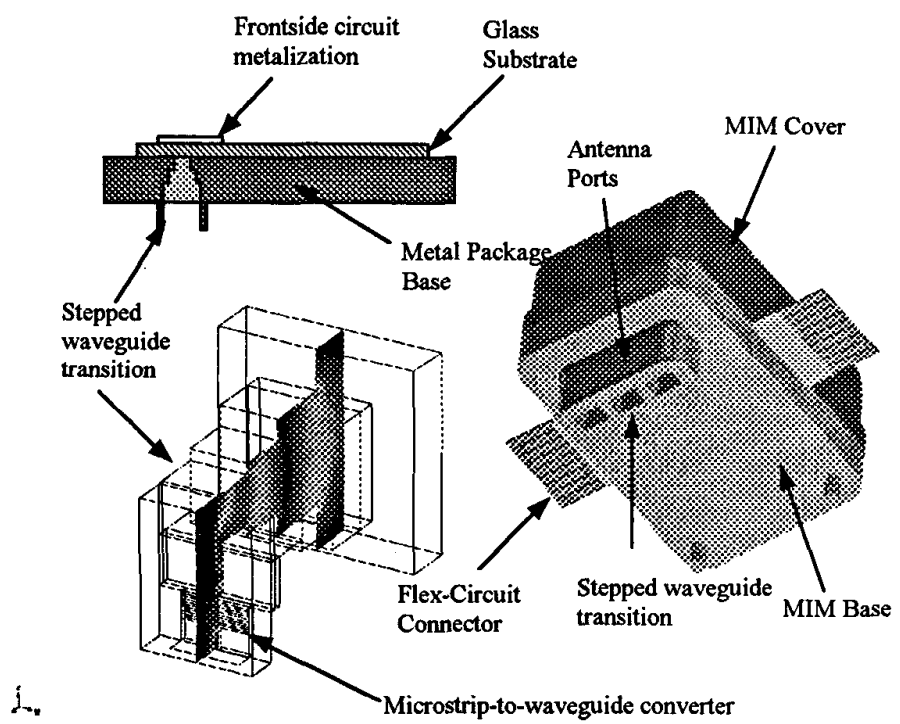

Figure 3 - Waveguide transition and package detail showing the concept of the 'drop-in' millimeter-wave module

The glass substrate mounted on the MIM base contains all of the millimeter-wave circuitry. An MIM cover is added to provide a self-contained dropin millimeter-wave module. All electrical interfaces between the module and the control and DSP circuits are maintained via the flex-cable. The module is mounted on the radar control board using a springclip attachment that allows for movement due to thermal effects of the mechanical interface. A common RF ground is established for the antenna by an interference-fit between the antenna ports and a corresponding recess on the antenna ground plane. The millimeter-wave module is easily integrated and removed from the radar module allowing for ease of testing and modular assembly.

The active devices and the dielectric puck may be mounted using conventional surface-mount pick-and-place technology. The discrete PHEMT devices used in the oscillator and amplifier are mounted upright and wire-bonded. The varactor diodes in the oscillator and frequency multipliers, and the Schottky diodes in the mixer are mounted using conventional flip-chip attach with a conductive silver loaded epoxy. Care must be taken in non-hermetic enclosures to ensure that the bias across the devices does not encourage silver-migration from the epoxy that compromises device reliability.

Although flip-chip attachment of discrete devices is a relatively trivial exercise, concerns with ground-plane coupling causing hybrid modes plus thermal management of heat dissipation makes the process more problematic for MMICs. In addition, the mismatch between the thermal coefficient of expansion of the MMIC and the substrate can require the use of an underfill material that can complicate circuit design. The MMIC circuits were thus mounted in the conventional upright position and connected using wire-bonds.

The use of compensated wire-bond interconnects that have an insertion loss of $<0.2 \mathrm{~dB}$ at $77 \mathrm{GHz}$ for bond-wires of length between $380 \mu \mathrm{m}$ 480um makes this compatible with the typical tolerance from an automated wire-bond machine [15].

\section{MODULE INTEGRATION AND CIRCUIT RESULTS}

The measured results of each of the circuits shown in Figure 2 are summarized in Table 1 . The module level results were obtained by mounting the transceiver module with a quasi-optical antenna from a commercially available ACC system. Figure 6 is an azimuth plot of the switched 3-beam antenna pattern for the transceiver module in transmit-mode. Each of 
the three beams provides approximately $3^{\circ}$ of azimuth coverage, whilst sidelobe levels are typically $-16 \mathrm{~dB}$.

\begin{tabular}{|c|c|}
\hline Description & Measured Performance \\
\hline $\begin{array}{l}\text { 19GHz DRO } \\
\text { and Amplifier }\end{array}$ & $\begin{array}{ll}- & \Delta f_{0} 120 \mathrm{MHz} \text { for } 8 \mathrm{~V} \text { tune voltage } \\
- & +14 \mathrm{dBm} \pm 0.75 \mathrm{~dB}\left(-40^{\circ} \mathrm{C} \text { to }+85^{\circ} \mathrm{C}\right) \\
- & \text { Frequency Drift } \sim 12 \mathrm{MHz} \\
- & \text { Switching speed }<5 \mathrm{nS}(10 \% \mathrm{DC} \text { to } 90 \% \mathrm{RF}) \\
- & \text { SSB Phase-Noise } \sim-92 \mathrm{dBc} / \mathrm{Hz} @ 100 \mathrm{kHz}\end{array}$ \\
\hline $\begin{array}{l}\text { 19-38GHz } \\
\text { Doubler }\end{array}$ & $\begin{array}{l}\text { - } 8 \mathrm{~dB} \text { conversion loss for }+10 \mathrm{dBm} \text { to } \\
+16 \mathrm{dBm} \text { input power level }(2.2 \mathrm{~V} \text { bias }) \\
\text { - } \quad \text { practically constant conversion loss over } \\
\text { temperature } \\
-\quad>15 \mathrm{~dB} \text { fundamental frequency rejection }\end{array}$ \\
\hline $\begin{array}{l}\text { 76GHz Mixer } \\
\text { Ref. [16] }\end{array}$ & $\begin{array}{l}\text { - } \quad<\mathrm{dB} \text { conversion loss with } 90 \% \text { yield for } \\
+8 \mathrm{dBm} \text { to }+16 \mathrm{dBm} \text { LO drive; IF = } \\
180 \mathrm{MHz}\end{array}$ \\
\hline PIN MMIC & $\begin{array}{ll} & \text { Insertion loss (Tx to antenna) } 3 \pm 0.5 \mathrm{~dB} \\
- & \text { Insertion loss (antenna to } \mathrm{Rx}) 2 \pm 0.5 \mathrm{~dB} \\
- & \text { Isolation (2-ports) }>23 \mathrm{~dB} ; 32 \mathrm{~dB} \text { typical } \\
\end{array}$ \\
\hline $\begin{array}{l}38-76 \mathrm{GHz} \\
\text { Doubler }\end{array}$ & $\begin{array}{l}\text { - } 12 \mathrm{~dB} \text { conversion loss for }+18 \mathrm{dBm} \text { to } \\
+23 \mathrm{dBm} \text { input power level } \\
\text { - practically constant conversion loss over } \\
\text { temperature }\end{array}$ \\
\hline
\end{tabular}

Table 1 - Measured performance of the components used in the millimeter-wave module

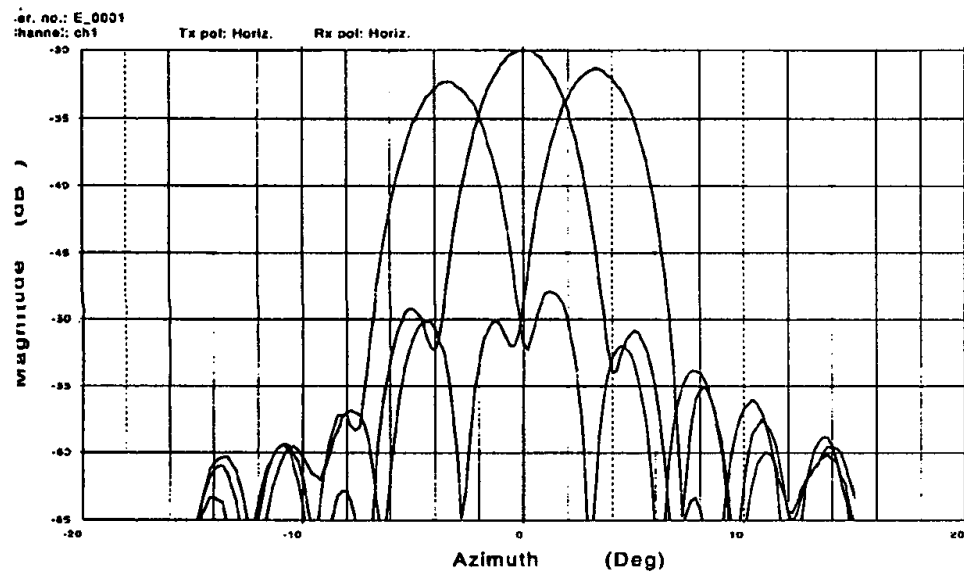

Figure 4-Aximuth beam pattem of the radar module in each of the three transmit beam states industry requires. The design philosophy behind this module represents a significant step forward in the ability to generate low-cost and high volume, consumer millimeter-wave products.

\section{ACKNOWLEDGEMENTS}

The authors would like to thank - amongst numerous others who made this first-pass design success possible - Dr. Gerry DiPiazza, Dr. Robert Egri, Marion Hines, Dr. J-P Lanteri, Dr. Clint Licqurish, and Da! Swanson for their advice, encouragement and support.

\section{REFERENCES}

[1] - JURGEN, R.K., (Editor), "Object Detection, Collisior' Warning and Avoidance Systems", Society of Automotive Engineers, 1998.

[2] - MENZEL, W. et al, "A 77GHz FMCW radar front-end with a lowprofile low-loss printed antenna", IEEE International Microwave Symposium (MTT-S), pp. 1485-1488, 1999.

[3] - MAYOCK, J. et al, "A wideband millimeter-wave front-end for automotive radar", IEEE MTT-S, pp. 1501-1504, 1999.

[4] - CAMIADE, M. et al, "Full MMIC millimeter-wave fiont-end for a 76.5GHz Adaptive Cruise Control Car Radar", IEEE MTI-S, pp. 14891492, 1999.

[5] - KERSSENBROCK, T.V. et al, "Novel $77 \mathrm{GHz}$ flip-chip sensor modules for Automotive radar applications". IEEE MTT-S, pp. 289-292, 1999.

[6] - LAMBERG, J.R. et al, “A compact, high perforniance $W$-band FMCW radar front-end based on MMIC technology", [EEE MTT-S, pp.1979-1800, 1999.

[7] - TESSMANN, A. et al, “A 77GHz GaAs pHEMT Transceive MMIC for Automotive Sensor Applications", IEEE GaAs IC S!mposium, pp. 207-210, 1999.

[8] - KONDOH, H. et al, "77GHz Fully-MMIC Autometive ForwardLooking Radar", IEEE GaAs IC Symposium, pp. 211-214, 1999.

[9] - MORENC, N.P., "MMICs for Automotive Radar .4pplications", IEEE MTT-S, pp.39-41, 1996.

[10] - TAKAHASHI, K et al, “An Advanced Millimeter-W'ave Flip-Chip IC Integrating Different Kinds of Active Devices", ]EEE MTT-S, pp.1619-1622, 1996

[11] - JAIN, N., "Designing Commercially Viable MM-Wizve Modules", To be presented IEEE MTT-S 2000.

[12] - CHINOY, P. et al, "Manufacture of Low-Loss Microwave Circuits Using HMIC Technology", 1994 IEEE MTT-S, pp. 1137-1 :140, 1994.

[13] - PERKO, R. et al, "MMIC vs. Hybrid: Glass N'icrowave ICs Rewrite the Rules", Microwave Journal, no.11, pp.67-78, 1988.

[14] - PUTNAM, J. et al, "A Monolithic GaAs PIN switch network for a $77 \mathrm{GHz}$ automotive collision warning radar", 1997 RFIC Symposium, pp. 225-228.

[15] - BUDKA, T.P., "Wide Bandwidth Millimeter-wizve Bondwire Interconnects", Submitted for Review to the IEEE Trans. on Microwave Theory and Techniques.

[16] - BUDKA, T.P. et al, "A 77GHz Mixer for Automotive Radar", Microwave Journal, pp. 132-134, January 2000.

\section{CONCLUSIONS}

A compact, complete transceiver module suitable for mm-wave $(76 \mathrm{GHz})$ vehicular radar applications has been described. All of the materials and assembly techniques used in the design are compatible with the low-cost objectives that the commercial mm-wave 